\title{
Opioid Release after High-Intensity Interval Training in Healthy Human Subjects
}

\author{
Tiina Saanijoki' , Lauri Tuominen ${ }^{1,2,3}$, Jetro J Tuulari', Lauri Nummenmaa ${ }^{1,4}$, Eveliina Arponen', \\ Kari Kalliokoski ${ }^{1}$ and Jussi Hirvonen*,1,5
}

I Turku PET Centre, University of Turku, Turku, Finland; ${ }^{2}$ Department of Psychiatry, Massachusetts General Hospital, Boston, MA, USA; ${ }^{3}$ Harvard Medical School, Boston, MA, USA; ${ }^{4}$ Department of Psychology, University of Turku, Turku, Finland; ${ }^{5}$ Department of Radiology, Turku University Hospital and University of Turku, Turku, Finland

\begin{abstract}
Central opioidergic mechanisms may modulate the positive effects of physical exercise such as mood elevation and stress reduction. How exercise intensity and concomitant effective changes affect central opioidergic responses is unknown. We studied the effects of acute physical exercise on the cerebral $\mu$-opioid receptors (MOR) of 22 healthy recreationally active males using positron emission tomography (PET) and the MOR-selective radioligand [ ' ' C]carfentanil. MOR binding was measured in three conditions on separate days: after a 60-min aerobic moderate-intensity exercise session, after a high-intensity interval training (HIIT) session, and after rest. Mood was measured repeatedly throughout the experiment. HIIT significantly decreased MOR binding selectively in the frontolimbic regions involved in pain, reward, and emotional processing (thalamus, insula, orbitofrontal cortex, hippocampus, and anterior cingulate cortex). Decreased binding correlated with increased negative emotionality. Moderate-intensity exercise did not change MOR binding, although increased euphoria correlated with decreased receptor binding. These observations, consistent with endogenous opioid release, highlight the role of the $\mu$-opioid system in mediating affective responses to high-intensity training as opposed to recreational moderate physical exercise. Neuropsychopharmacology (2018) 43, 246-254; doi:I0.1038/npp.2017.148; published online 16 August 2017
\end{abstract}

\section{INTRODUCTION}

Physical exercise improves mental health by elevating mood (Kanning and Schlicht, 2010; Yeung, 1996) and by reducing stress and symptoms of depression and anxiety (Scully et al, 1998). Exercise also improves cognitive performance and brain plasticity (Aberg et al, 2009; Colcombe et al, 2004), and has well-known cardiovascular and metabolic benefits. Previous research has confirmed that enjoyment is an important incentive for engaging in leisure-time physical activity (Aaltonen et al, 2012). Many people report pleasurable sensations and improved mood after physical exercise (Mazzeschi et al, 2014) and some even describe euphoric feelings ('runner's high') in response to prolonged physical exercise (Dietrich and McDaniel, 2004; Morgan, 1985).

Exercise intensity is one of the key modulators of exerciseinduced pain and pleasure (Oliveira et al, 2015). Exercise of low and moderate intensity is associated with positive mood changes, whereas higher intensities tend to impair affect both during and immediately after exercise (Ekkekakis et al, 2011). The affective characteristics of interval exercise are less well understood, but have recently gained attention due

* Correspondence: Dr J Hirvonen, Department of Radiology and Turku PET Centre, University of Turku, Kiinamyllynkatu 4-8, Turku FIN-20520, Finland, Tel: +358 50585 8865, Fax: +358 2313 7908,

E-mail: jussi.hirvonen@utu.fi

Received 17 January 20 17; revised 10 July 20 17; accepted II July 20 I7; accepted article preview online 19 July 2017 to the growing popularity of high-intensity interval training (HIIT). HIIT is superior to moderate-intensity continuous training (MICT) in improving several markers of cardiovascular fitness with reduced training time commitment (Milanović et al, 2015; Ramos et al, 2015; Weston et al, 2014). HIIT consists of brief (5-150 s) but intense bursts of activity interspersed with short periods of rest or active recovery, and typically elicits more negative mood responses and higher perceived exertion and pain than the more traditional MICT (Decker and Ekkekakis, 2017; Jung et al, 2014; Saanijoki et al, 2015). This may not, however, be the case for physically fit individuals who may experience improved mood and enjoyment also after HIIT (Bartlett et al, 2011).

The neurobiological mechanisms underlying exerciseinduced affective responses are not known. The most commonly adopted theory on physical exercise induced euphoria is the 'endorphin hypothesis', which ascribes the positive mood changes following exercise to an increased release of $\beta$-endorphins (Morgan, 1985; Yeung, 1996). The level of plasma $\beta$-endorphin is usually elevated during intense exercise (Goldfarb and Jamurtas, 1997), but a plausible link between circulating endorphin concentrations and mood responses to acute exercise has not been established (Dishman and O'Connor, 2009).

The endogenous mesolimbic opioid system contributes to the rewarding effects of external factors like food, drugs, and social interaction (Henriksen and Willoch, 2008; Trezza et al, 
2011). Because endogenous opioids modulate affect (positive and negative) (see review in Nummenmaa and Tuominen, in press) and pain (Zubieta et al, 2001), exercise-induced affective responses might also be attributed to the brain opioid system. Of the three types of opioid receptors $(\mu-, \delta$-, and $\kappa$-receptors), the $\mu$-opioid receptors (MOR) mediate the effects of endogenous opioids (eg, $\beta$-endorphins) and of exogenous opioid agonists and antagonists, and are thus responsible for mediating positive reward and euphoria (Chartoff and Connery, 2014).

In humans, endogenous opioid release can be examined with positron emission tomography (PET) by changes in the binding of $\left[{ }^{11} \mathrm{C}\right]$ carfentanil, a highly selective high-affinity agonist radioligand for MOR (Colasanti et al, 2012; Mick et al, 2014, 2016; Titeler et al, 1989). Although a previous PET study linked 'runner's high' (euphoria after $2 \mathrm{~h}$ of running) with a decrease in the binding of the non-selective opioid receptor radioligand $\left[{ }^{18} \mathrm{~F}\right] \mathrm{FDPN}$ (Boecker et al, 2008), the relation between MORs and physical exercise in humans is not known.

In this study, we measured the effects of different types of acute physical exercise on MOR availability in 22 healthy men using PET and $\left[{ }^{11} \mathrm{C}\right]$ carfentanil. The participants were scanned after rest and after $60 \mathrm{~min}$ of MICT on cycle ergometer, 12 of them additionally after HIIT, in a counterbalanced order. We used a classic form of recreational $60 \mathrm{~min}$ instead of prolonged sessions of aerobic exercise traditionally associated with 'runner's high' in an attempt to examine the typical positive mood changes associated with shorter exercise challenges, and to make the results applicable to the population. In addition, we employed HIIT, a popular and time saving but strenuous method in improving cardiovascular fitness. We hypothesized that physical exercise leads to decreased MOR binding consistent with endogenous opioid release and that HIIT is even more effective in this respect, and that MOR binding correlates with exercise-induced positive mood changes.

\section{MATERIALS AND METHODS}

\section{Ethics}

The study was conducted at the Turku PET Centre, University of Turku and Turku University Hospital (Turku, Finland) and followed the principles of the Declaration of Helsinki. The Ethics Committee of the Hospital District of South-West Finland approved the research protocol. The purpose and potential risks of the study were explained to the subjects each of whom gave written informed consent. This study was registered at clinicaltrials.gov under the number NCT02615756.

\section{Subjects}

In total 22 healthy men (age $26.1 \pm 4.9$ years, range $21-36$ years) with a variable exercise background participated in the study (Table 1). The inclusion criteria were male sex, age 1865 years, and body mass index below $27 \mathrm{~kg} \mathrm{~m}^{-2}$. The exclusion criteria were a history of or current neurological or psychiatric disease, use of tobacco products or medication affecting the central nervous system, current or past excessive alcohol or substance abuse, any chronic illness resulting in
Table I Study Participants $(N=22)$

Mean (SD)

\begin{tabular}{lc}
\hline Age (years) & $26.1(4.9)$ \\
BMI $\left(\mathrm{kg} / \mathrm{m}^{2}\right)$ & $23.4(1.7)$ \\
Physical activity (min/week) & $276(\mathrm{I} 28)$ \\
$\mathrm{VO}_{2 \max }(\mathrm{ml} / \mathrm{kg} / \mathrm{min})$ & $48.9(6.2)$ \\
Work load $_{\max }(\mathrm{W})$ & $303(50)$ \\
Heart rate & $195(7)$ \\
\hline
\end{tabular}

Abbreviations: $\mathrm{BMI}$, body mass index; $\mathrm{VO}_{2 \max }$, maximal oxygen uptake.

disability in daily life, excessive or competitive athletics not consistent with common exercising habits, claustrophobia, and the presence of any ferromagnetic objects that would contraindicate magnetic resonance imaging. Laboratory tests, urinanalysis, and an ECG were obtained to assess health and the absence of psychoactive drugs.

\section{Maximal Exercise Test}

To determine the individual workload for the MICT exercise task, the subjects performed a maximal aerobic exercise test on a bicycle ergometer (Ergoline 800 s, VIASYS Healthcare, Germany) starting at $40 \mathrm{~W}$ and followed by $30 \mathrm{~W}$ increments every 2 min until volitional exhaustion. Ventilation and gas exchange were measured (Jaeger Oxycon Pro, VIASYS Healthcare, Germany) and reported as the mean value per minute. The highest 1-min value of oxygen consumption was expressed as the $\mathrm{VO}_{2 \max }$. The maximal workload ( $\operatorname{Load}_{\max }$ ) was calculated as the average workload during the last two minutes of the test and used as a measure of maximal performance. Metabolic thresholds were determined from the blood lactate concentration measured from capillary samples taken repeatedly during the test. The aerobic threshold corresponded the workload at which the blood lactate rose above the baseline level, and the anaerobic threshold the workload at which blood lactate began to accumulate rapidly.

\section{Experimental Design}

In total 10 participants underwent two consecutive PET studies -one at rest and another after completion of a MICT session. Twelve other participants underwent three PET studies-after MICT, after HIIT, and after rest (Supplementary Figure S1). A bolus injection of $\left[{ }^{11} \mathrm{C}\right]$ carfentanil was used. The participants fasted for $3 \mathrm{~h}$ before studies and were instructed to discontinue the use of caffeine containing products and not perform any physical exercise $24 \mathrm{~h}$ before the PET studies. For all participants, the order of the PET studies was randomized and all studies took part on separate days.

The MICT session consisted of $60 \mathrm{~min}$ of continuous aerobic cycling (Tunturi E85, Tunturi Fitness, Almere, The Netherlands) at workload in the middle between aerobic and anaerobic thresholds predetermined individually by the maximal exercise test (mean workload $164 \pm 42 \mathrm{~W} ; 54 \pm 7 \%$

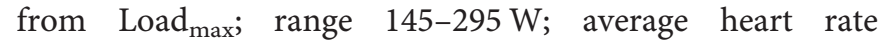
during exercise $143 \pm 15$ beats per min; $74 \pm 7 \%$ from maximal heart rate). All participants performed the MICT 
session successfully. Because of an unexpected delay with radiotracer supply, one of the participants had a slightly longer MICT session (77 min rather than the programmed $60 \mathrm{~min})$.

The HIIT session consisted of $4 \mathrm{~min}$ of warm-up and five all-out cycling efforts (Monark Ergomedic 894E; Monark, Vansbro, Sweden) with $4 \mathrm{~min}$ of recovery, during which participants remained still or did unloaded cycling. Each sprint started with a few seconds of acceleration to maximal cadence, followed by a sudden increase of the load (7.5\% of body weight) and maximal cycling for $30 \mathrm{~s}$. Participants were familiarized with the HIIT protocol before HIIT session. Two participants could perform only three $30 \mathrm{~s}$ sprints: one was too exhausted and experienced knee pain, the other vomited after three bouts. Nevertheless, the data from these studies was included in the data analysis.

Heart rate was monitored during exercise (RS800CX; Polar Electro Ltd., Kempele, Finland). The blood lactate concentration was measured from venous samples before and within $1 \mathrm{~min}$ after completion of training (Lactate Pro; Arkray KDK, Kyoto, Japan). Music, television, or other technical devices were not available to the subjects during exercise. PET scanning began within 15-37 min after the completion of the exercise session, concomitantly with the administration of the radiotracer.

During the MICT and HIIT sessions the participants' subjective degree of exertion was assessed with Borg's Rating of Perceived Exertion (RPE) 6-20 scale and their subjective feelings of emotional valence (pleasant versus unpleasant) and arousal (calm versus excited) were assessed with the SelfAssessment Manikin (SAM) rating scale (Bradley and Lang, 1994) (Supplementary Figure S2). The RPE and SAM scales were administrated before, and at 15, 30, 45 and $60 \mathrm{~min}$ during MICT, and before HIIT and within $5 \mathrm{~s}$ after each $30 \mathrm{~s}$ sprint. Subjective feelings of pleasant versus unpleasant emotions were measured using the Positive and Negative Affect Schedule (PANAS) (Watson et al, 1988) and a visual analog scale (VAS; separate scales for tension, irritation, pain, exhaustion, satisfaction, motivation to exercise, euphoria, and energy) before and within $3 \mathrm{~min}$ after the end of each training session. After the HIIT session, three of the participants vomited and filled the questionnaires within 15 min after exercise completion. Euphoria and energy were added in the middle of the study, thus only 15 participants completed full VAS questionnaire.

On the day of the baseline study, the participants rested for $60 \mathrm{~min}$ before the studies without music, television, or mobile entertaining devices. PANAS and VAS were administrated before and after the scans.

\section{Radiotracer Synthesis and PET Data Acquisition and Analysis}

$\left[{ }^{11} \mathrm{C}\right]$ Carfentanil was synthesized by ${ }^{11} \mathrm{C}$-methylation of desmethyl carfentanil (sodium salt) with $\left[{ }^{11} \mathrm{C}\right]$ methyl triflate prepared from cyclotron-produced $\left[{ }^{11} \mathrm{C}\right]$ methane. The synthesis was performed according to the procedure previously published (Hirvonen et al, 2009) with minor modifications. An intravenous catheter was placed in the left arm of each study participant for blood sampling and radiotracer injection. The radioligand was administered at tracer doses with no expected pharmacological side effects. The specific radioactivity and the injected mass of the radioligand did not differ between the scans (Supplementary Table S1). After an intravenous injection of $\left[{ }^{11} \mathrm{C}\right]$ carfentanil $(257 \pm 12 \mathrm{MBq}$, range $224-295 \mathrm{MBq}$, injected mass $0.26 \pm 0.17 \mu \mathrm{g}$, specific radioactivity $507 \pm 259 \mathrm{MBq} / \mathrm{nmol}$ ), radioactivity in the brain was measured with a Philips Ingenuity PET/MR scanner for $51 \mathrm{~min}$, using 13 frames, with in-plane resolution of $3.75 \mathrm{~mm}$. A T1- weighted TR $25 \mathrm{~ms}$, TE $4.6 \mathrm{~ms}$, flip angle $30^{\circ}$, scan time $376 \mathrm{~s}$ MR images $\left(1 \mathrm{~mm}^{3}\right.$ voxel size) were acquired for anatomical reference. Radioactivity data acquisition was started concomitantly with the injection of the radiotracer.

To correct for head motion all the volumes of the dynamic PET scans were realigned to each other. A T1-weighted MR image was coregistered with the summed PET image and the occipital cortex was manually defined on the coregistered T1 image using the PMOD 3.3 software (PMOD Technologies, Zürich, Switzerland). Receptor availability was expressed in terms of $B P_{\mathrm{ND}}$, which is the ratio between specific and nondisplaceable binding in the brain. A simplified reference tissue model (SRTM) was used to obtain non-displaceable binding potential estimates voxel-wise using occipital time activity curve as reference tissue input (Gunn et al, 1997). This outcome measure is not confounded by changes or differences in peripheral distribution. Specific binding of $\left[{ }^{11} \mathrm{C}\right]$ carfentanil is unaffected by changes in cerebral blood flow (Endres et al, 2003; Frost et al, 1989; Liberzon et al, 2002). The resulting parametric $B P_{\mathrm{ND}}$ images were normalized to the Montreal Neurological Institute (MNI) space using deformation fields obtained by segmenting the T1-weighted images. Finally, normalized parametric images were smoothed with a Gaussian kernel of $7 \mathrm{~mm}$ full-width half-maximum. All preprocessing steps were carried out using the SPM8 software (www.fil.ion.ucl.ac.uk/spm/) run on Matlab R2012a (MathWorks), except for simplified reference tissue modeling, which was done using PMOD. Because of technical problems with the PET scanner, the PET-data following one MICT and one HIIT were subsequently found to be invalid and were excluded from the analysis.

\section{Statistical Analyses}

Within subject effects of MICT on MOR availability were tested with the paired $t$ test for the whole group, since every subject completed rest and MICT scans $(n=21$, age 25.1 (8.6) years). The within subject effects of MICT and HIIT on MOR availability were tested with repeated measures oneway ANOVA for the subset of participants who completed all three studies ( $n=11$, age 24.8 (9.1) years) (Supplementary Figure S1). The calculations were performed using the SPM8 software (www.fil.ion.ucl.ac.uk/spm/) running on Matlab R2012a (MathWorks). The threshold for statistically significant differences was set at $p<0.05$ for all assessments, false discovery rate corrected at cluster level. The Marsbar toolbox for SPM (http://marsbar.sourceforge.net/) was used to extract mean $B P_{\mathrm{ND}}$ estimates from each activation cluster observed in the full-volume analysis.

The effects of physical exercise on RPE, valence and arousal measured during exercise, and positive and negative affect and VAS items measured before and after exercise, were analyzed using ANOVA for the participants who 
performed both exercise modes (MICT and HIIT, $n=12$ ) with IBM SPSS Statistics 21 for Mac OS X (IBM Corp., Chicago, IL, USA). To ensure that MICT-induced affective responses were similar between the group that performed only MICT $(n=10)$ and the group that performed both MICT and HIIT $(n=12)$, another set of general linear model analyses were performed using condition (pre/post) as a within-subject factor and group (only MICT; or both MICT and HIIT) as a between-subject factor. The associations of exercise-induced changes in mood responses and changes in MOR binding were assessed using an exploratory wholebrain analysis with statistical threshold set at $p<0.05$, false discovery rate corrected at cluster level.

\section{RESULTS}

\section{Effects of Exercise on MOR Binding}

After HIIT, $\left[{ }^{11} \mathrm{C}\right]$ carfentanil $B P_{\mathrm{ND}}$ values were significantly lower in widespread regions of the brain than after rest or MICT conditions according to full-brain voxel-based analysis (Figure 1). This was the case for cortical regions (prefrontal cortex, anterior cingulate cortex, and insula) and subcortical brain regions (hippocampus, thalamus, amygdala, ventral striatum, periaqueductal gray matter in the brainstem, and cerebellum). The average change of $\left[{ }^{11} \mathrm{C}\right]$ carfentanil $B P_{\mathrm{ND}}$ was $-19 \pm 14 \%$ (range $-50 \%$ to $0 \%$ ) (Figure 2). HIIT did not induce increases of $\left[{ }^{11} \mathrm{C}\right]$ carfentanil binding. In contrast, $\left[{ }^{11} \mathrm{C}\right]$ carfentanil $B P_{\mathrm{ND}}$ values were not significantly affected after MICT compared with rest, neither for the whole group $(n=21)$ using paired $t$ test nor in the repeated measures one way ANOVA for the subset of participants who underwent all three scans $(n=11)$. The results were the same even after controlling for scan order (whole brain analysis $p<0.05$ ).

Regional time-activity curves suggested that physical exercise changed brain uptake in a global fashion both in target and reference regions. This would be consistent with a change in the arterial input function, perhaps due to changes in the peripheral distribution after exercise (Figure $3 \mathrm{a}$ and $\mathrm{b}$ ). Nevertheless, regional radioactivity concentration ratios of target and reference regions suggested lower specific binding of $\left[{ }^{11} \mathrm{C}\right]$ carfentanil after HIIT, despite any such changes in radioligand delivery (Figure $3 \mathrm{c}$ ). To further investigate the occurrence of changes in radioligand delivery to the target, we analyzed data from defined anatomical regions of interest, which we defined manually: thalamus, ventral striatum, dorsal anterior cingulate cortex, dorsolateral prefrontal cortex, and insula. For this, we used SRTM as implemented in PMOD. We found that $B P_{\mathrm{ND}}$ was significantly decreased after HIIT $(n=11)$ in the dorsal anterior cingulate cortex $(-10 \%, p=0.003)$ and ventral striatum $(-8 \%, p=0.044)$, but unchanged after MICT $(n=21$; all $p>0.1)$. This is in agreement with the full-brain voxel-based analysis results. In addition, we found that the estimate of radioligand delivery in the target region relative to the reference region (R1) was also slightly reduced after HIIT in the dorsal anterior cingulate cortex $(-6 \%, p=0.040)$ and insula $(-8 \%, p=0.028)$, whereas the efflux constant from target tissue to plasma $\left(\mathrm{k}_{2}\right)$ was reduced in the ventral striatum $(-10 \%, p=0.040)$ (Supplementary Table S2).

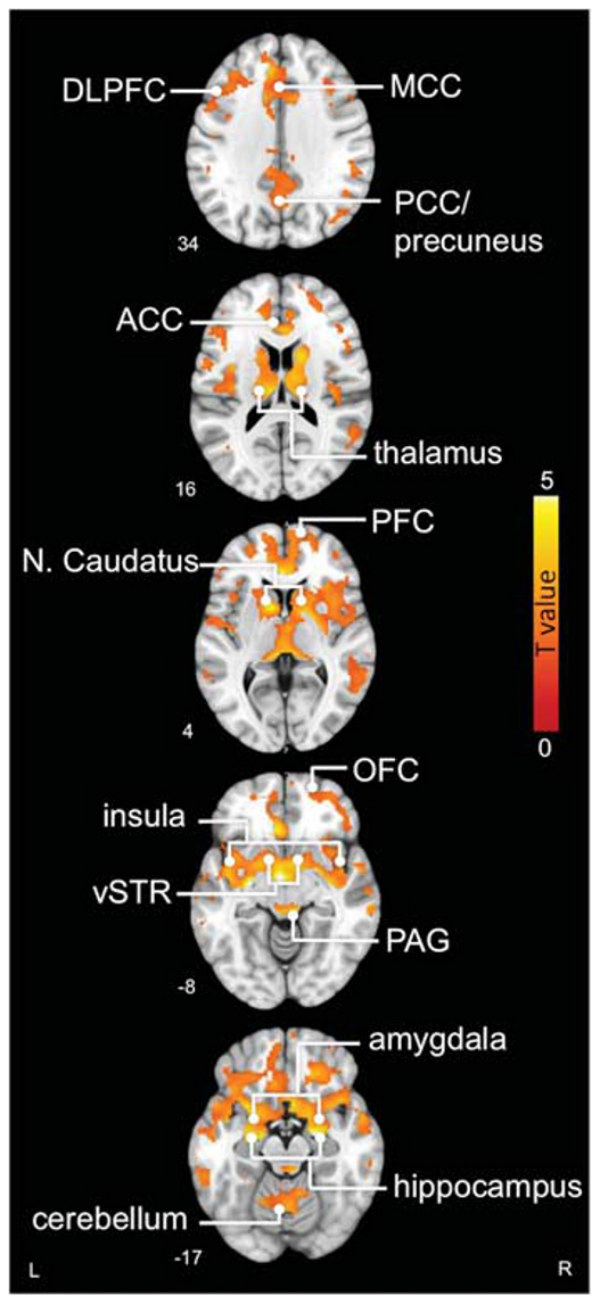

Figure I Brain regions showing significantly decreased MOR availability after HIIT in comparison with rest. The data are thresholded at $p<0.05$, false discovery rate corrected. Peak voxels are located in the left hippocampus, coordinates $-26,-10,-12 \mathrm{~mm}(x, y$, and $z$, respectively, in $\mathrm{MNI}$ space), cluster size I 1098 voxels, $T=6.3, Z=4.99$; and in the left cerebellum crus $\|$, coordinates $-14,-84,-28 \mathrm{~mm}$, cluster size 1922 voxels, $T=3.83, Z=3.43$. ACC, anterior cingulate cortex; DLPFC, dorsolateral prefrontal cortex; L, left; MCC, middle cingulate cortex; $\mathrm{N}$, nucleus; OFC, orbitofrontal cortex; PAG, periaqueductal gray matter; PCC, posterior cingulate cortex; PFC, prefrontal cortex; R, right; VSTR, ventral striatum.

\section{Effects of Exercise on Emotional State}

In the subset of participants who performed both exercise modes $(n=12)$, HIIT increased perceived exertion and arousal more and decreased affective valence more than MICT during exercise, $F_{1,11}=31.71, p<0.001 ; F_{1,11}=6.44$, $p=0.028$; and $F_{1,11}=13.44, p=0.004$, respectively. Participants experienced more positive affect after MICT than after HIIT, $F_{1,11}=10.01, p=0.009$, and more negative affect after HIIT than after MICT, $F_{1,11}=29.37, p<0.001$. Exhaustion ratings were higher after HIIT than after MICT, $F_{1,11}=6.92$, $p=0.023$. Tension ratings were higher under HIIT than MICT conditions, but the pre- and post-exercise ratings did not differ significantly from each other, $F_{1,11}=13.49$, $p=0.004$. Satisfaction ratings increased after MICT and decreased after HIIT, $F_{1,11}=7.60, p=0.019$. Pain ratings 


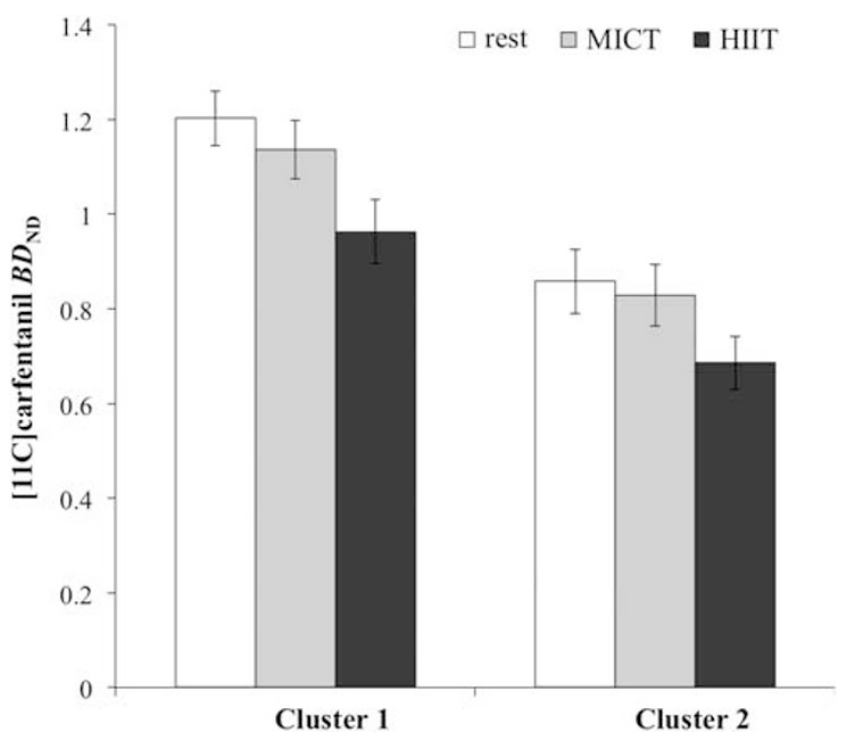

Figure 2 Means and standard errors of the means of $\left[{ }^{1} \mathrm{C}\right]$ carfentanil $B P_{N D}$ in both statistically significant clusters in each condition. Data include subjects who performed all three scans (rest, MICT, HIIT; $n=I I$ ) and are shown for visualization purposes only, statistical inference is based on the full-volume SPM analysis.

were higher after exercise, but not differently so between MICT and HIIT, $F_{1,11}=9.62, p=0.01$. Motivation to exercise was lower under HIIT than MICT conditions and there were no differences in ratings between before and after exercise, $F_{1,11}=10.14, p=0.009$. Irritation ratings were higher after HIIT than after MICT, $F_{1,11}=10.91, p=0.007$. Euphoria ratings were higher in MICT than in HIT condition, $F_{1,11}=4.74, p=0.052$, and higher after exercise, $F_{1,11}=6.00$, $p=0.032$. Feeling of energy was higher after MICT than after HIIT, $F_{1,11}=14.98, p=0.003$. Lactate was higher after HIIT than after MICT, $F_{1,11}=923.62, p<0.001$. There were no differences in MICT-induced affective responses between the subset of participants who performed only MICT and the subset of participants who performed both MICT and HIIT (all $p>0.05$ ).

To examine if exercise-induced changes in mood responses are associated with changes in MOR binding, exploratory whole-brain analysis was used. After MICT, increased euphoria after exercise correlated with decreased $B P_{\mathrm{ND}}$ in the dorsal prefrontal cortex $(r=0.81)$ and precuneus $(r=0.80)$ (Figure 4a). Decreased valence during MICT was positively associated with decreased $B P_{\mathrm{ND}}$ in the orbitofrontal $(r=0.63)$, and a perceived exertion increase was associated with a reduction in $B P_{\mathrm{ND}}$ in the frontal $(r=0.77)$ and parietal regions $(r=0.71)$ of the brain. After HIIT, an increase in negative affect (Figure $4 \mathrm{~b}$ ) and tension correlated with decreased $B P_{\mathrm{ND}}$ in the frontal cortex $(r=0.75$ and $r=0.90$, respectively), and an increase in dissatisfaction correlated with decreased $B P_{\mathrm{ND}}$ in the thalamus $(r=0.76)$. No inverse correlations were recorded.

\section{DISCUSSION}

Our results indicate that brain opioid action has a previously unrecognized role in association with acute physical exercise. We showed that the exercise intensity modulates MOR
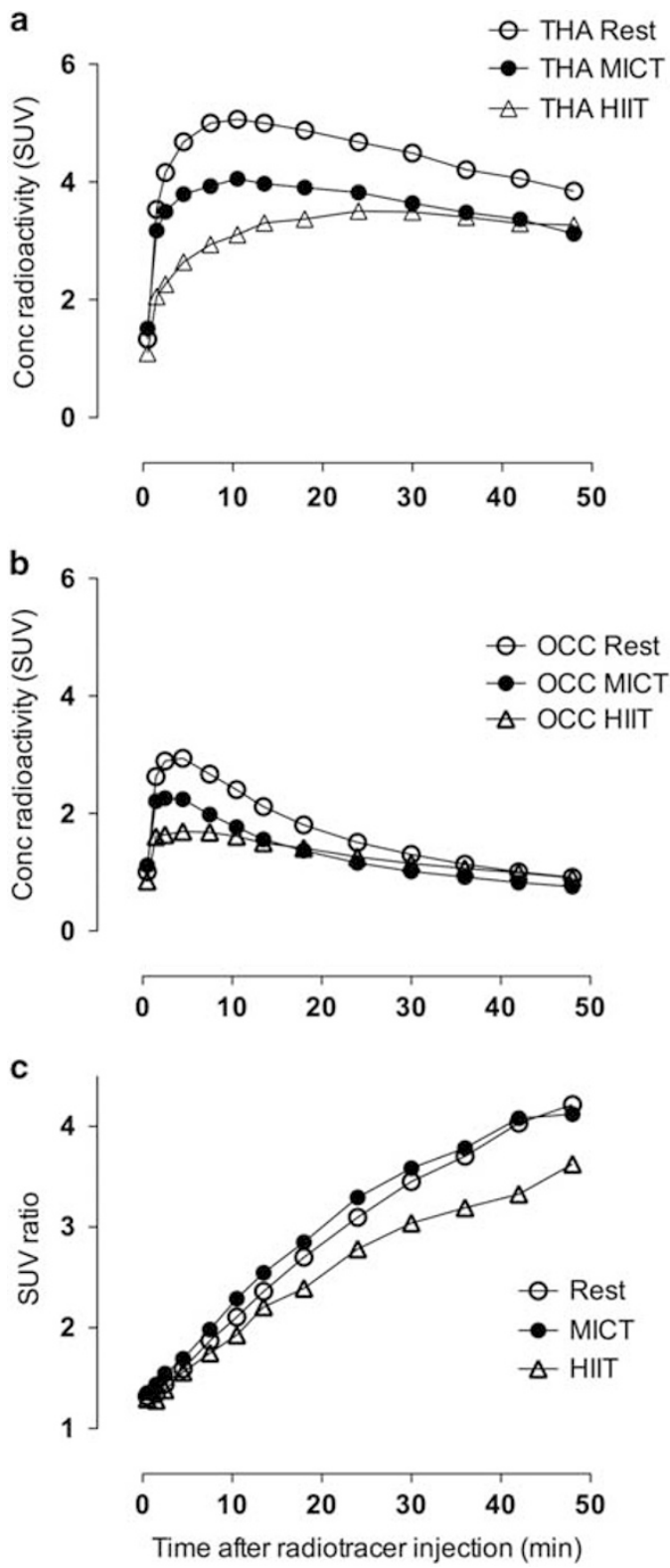

Figure 3 Means of regional radioactivity concentrations over time in the thalamus (THA), a target region (a), and occipital cortex (OCC), the reference region (b) after injection of [ I I C]carfentanil. Although changes in both target and reference regions after HIIT suggests altered arterial input function, possibly due to peripheral effects, radioactivity concentration ratios between target and reference regions clearly suggest lower specific uptake after HIIT (c).

activation and the concomitant changes in affective state. High-intensity exercise elicits endogenous opioid release, as showed by significantly decreased MOR availability in widespread brain regions where pain, mood, and affective states are processed, ie, the thalamus, anterior cingulate, orbitofrontal and insular cortices. Moderate-intensity exercise did not induce any net change in MOR availability, but it did improve the positive affect, satisfaction and euphoria, and changes in these variables correlated with change in MOR binding. In contrast, high-intensity exercise resulted in increased negative affect, exhaustion, and irritation, and loss of energy. This pattern of results suggests that MOR may 
a
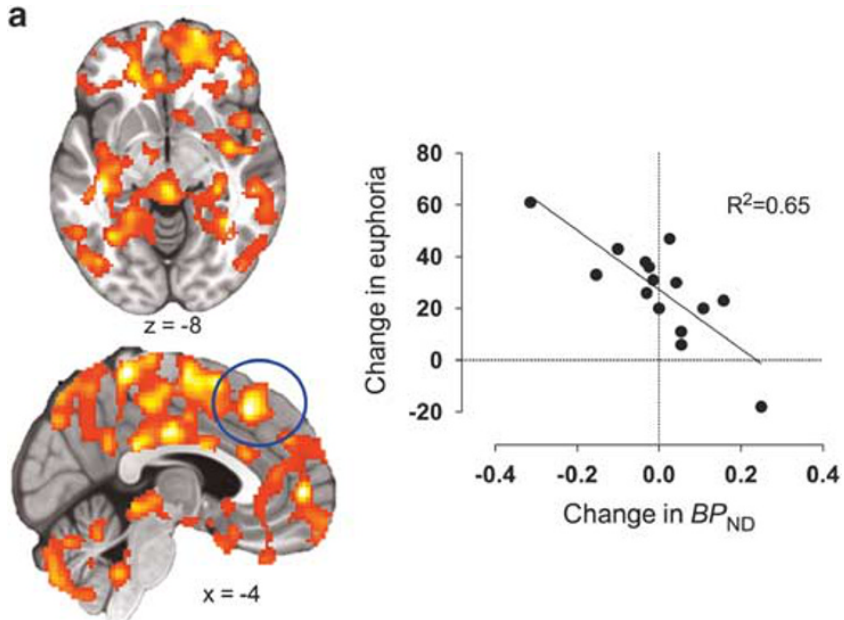

b
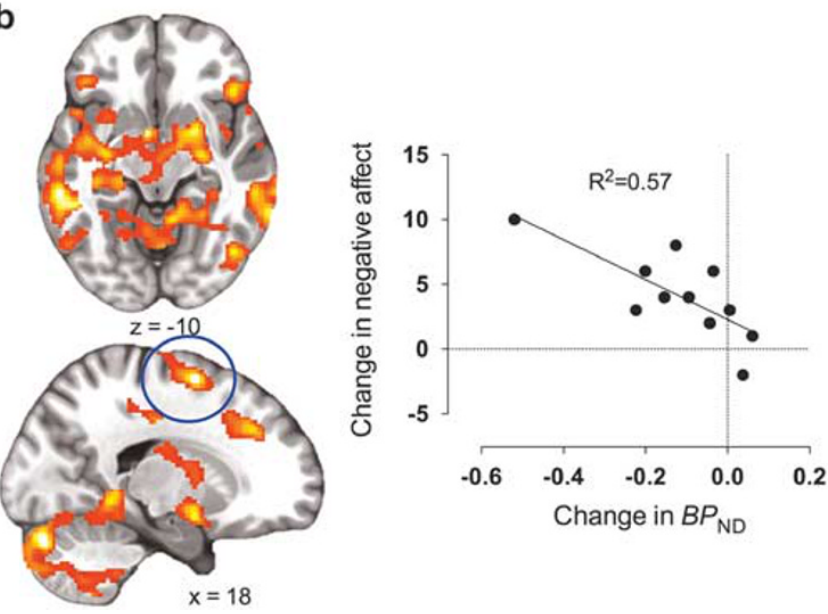

Figure 4 Whole brain exploratory analysis revealed that changes in $\left[{ }^{\prime \prime} \mathrm{C}\right]$ carfentanil $B P_{N D}$ predicts increased euphoria after MICT (a) and increased negative affect after HIIT (b). Circles denote the clusters where BP ND changes are shown against euphoria (a) and negative affect (b) in the scatterplots. Cook's distance for all observations is $<$ I suggesting that no single data point or their removal significantly biases the correlation. Peak voxels for euphoria are located in the dorsal prefrontal cortex, coordinates $6,26,46 \mathrm{~mm}$ ( $x, y$, and $z$, respectively, in MNI space), cluster size II 039 voxels, $T=6.11, Z=4.12$; in the medial prefrontal cortex, coordinates 4, 56, $2 \mathrm{~mm}$, cluster size 988 voxels, $T=5.43, Z=3.86$; and in the precuneus, coordinates $8,-74,40 \mathrm{~mm}$, cluster size 1499 voxels, $T=5.26, Z=3.78$. Peak voxel for negative affect is located in the frontal cortex, coordinates 20 , 2, $62 \mathrm{~mm}$, cluster size 31066 voxels, $T=6.7 \mathrm{I}, Z=3.92$.

have a dual role at different levels of physical exercise: modulation of positive emotionality at low-moderate intensity exercise and modulation of negative emotionality at high-intensity exercise.

Decreased MOR availability after HIIT compared with availability after rest is consistent with increased endogenous opioid release (Colasanti et al, 2012; Mick et al, 2014, 2016). In a previous study, increased opioid activation was demonstrated with the non-selective opioid receptor radioligand $\left[{ }^{18} \mathrm{~F}\right] \mathrm{FDPN}$ after $2 \mathrm{~h}$ of running and concomitant sensations of euphoria in experienced endurance athletes (Boecker et al, 2008). $\left[{ }^{18} \mathrm{~F}\right] \mathrm{FDPN}$ binding, however, does not differentiate between the opioid receptor subtypes and thus changes in $\left[{ }^{18} \mathrm{~F}\right] \mathrm{FDPN}$ may reflect a combination of changes in several receptor subtypes. Given that the MOR is the receptor subtype most relevant in mediating analgesia and reward, our finding of decreased $\left[{ }^{11} \mathrm{C}\right]$ carfentanil $B P_{\mathrm{ND}}$ specifically reflects the release of MOR-binding opioids such as endorphins and enkephalins, and thus more closely defines opioid action in response to exercise. In contrast to previous findings after $2 \mathrm{~h}$ of running, we did not find activation of MOR function after one hour of indoor cycling, yet it increased positive affect, satisfaction, and energy. Although there was no net change in MOR binding, we found that decreased binding predicted increased euphoria, suggesting some level of modulation by MOR function. These milder mood improvements, in comparison with the 'runner's high' associated with prolonged exercise such as marathon running, are typically reported to occur in response to low to moderate-intensity exercise and physical activity in general (Hall et al, 2002; Kilpatrick et al, 2007). Significant dilution of the $B P_{\mathrm{ND}}$ effect by the time between the exercise and the PET study seems unlikely, as decreased $B P_{\mathrm{ND}}$ persists for 20-65 min after pain stimulation (Scott et al, 2007). Mood elevation after moderate intensity exercise is probably also modulated by other neural factors and neurotransmitter systems, such as the endocannabinoid system (Fuss et al, 2015).

Decreased MOR availability following HIIT, but not MICT indicates that endogenous opioid action at the MOR sites in response to physical exercise may be more important for more strenuous exercise regimens. This hypothesis is supported by findings in healthy subjects that blocking MOR with naloxone decreases maximal cycle ergometry performance mainly through increased perceived exertion rather than by physiological limits (Sgherza et al, 2002). Peripheral $\beta$-endorphin levels have also been found to rise in response to high-intensity or prolonged exercise, but not in response to low or moderate-intensity exercise (Schwarz and Kindermann, 1992). This implies that endogenous opioid activation after high-intensity exercise is related to mechanisms of antinociception and stress relief. An acute bout of HIIT induces greater physiological (Wahl et al, 2013) and emotional stress and pain than aerobic exercise (Saanijoki et al, 2015), and may well result in endogenous opioid release and MOR activation, which not only promotes analgesia, but also regulates the stress response by modulating behavior and responses of the endocrine and autonomic nervous system. The lesser demands of $1 \mathrm{~h}$ of aerobic exercise may not result in such persistent stimulation of the MOR system.

We recorded significant decreases in MOR availability specifically in regions related to processing pain and analgesia: the thalamus, anterior cingulate cortex, insula, prefrontal cortex, basal ganglia, and periaqueductal gray matter (Duerden and Albanese, 2013; Zubieta et al, 2001). These regions are also primary for reward and emotions (Haber and Knutson, 2010) and process both positive and negative affect (Saarimäki et al, 2016). We found diverse associations between exercise-induced mood changes and changes in MOR availability in these regions, and these associations are consistent with a role of the endogenous opioid system for pain and pleasure (Leknes and Tracey, 2008). Although unequal sample sizes may confound the comparison between MICT and HIIT correlations, a more spatially widespread correlation was seen between increased euphoria after MICT than between increased negative affect 
after HIIT (Figure 4). There was a stronger correlation in the prefrontal and anterior cingulate cortex in MICT than in HIIT analysis, maybe due to the importance of these brain regions with regard to reward processing (Haber and Knutson, 2010). Although there were no correlations between MOR availability and pain ratings related to HIIT, the increased pain associated with HIIT may contribute to MOR activation. MOR activation in the anterior cingulate cortex and thalamus is associated with attenuation of the affective component of pain (Zubieta et al, 2001), and this could contribute also to tolerance of high-intensity exercise. But ultimately, we cannot separate the specific contributions from pain, analgesia, and negative emotionality to the outcomes in this study setting, as all of these functions involve MOR activation in these brain regions.

Previous animal studies have not yielded conclusive data regarding MOR function after physical exercise. A recent autoradiography study using $\left[{ }^{3} \mathrm{H}\right] \mathrm{DAMGO}$, a selective MOR agonist, showed increased MOR binding in several cortical regions after a few repeated low-intensity exercise sessions (7 days, $1 \mathrm{~h}$ per day) in rats (Arida et al, 2015). Another study reported increased MOR expression in rat hippocampal formation in response to acute, moderate-intensity, voluntary, and forced exercise (de Oliveira et al, 2010). The findings after habitual exercise are mixed, as chronic prolonged exercise results in decreased MOR expression (de Oliveira et al, 2010) and increased, as well as decreased MOR availability (Arida et al, 2015) in rat hippocampal formation. Rats selectively bred for high voluntary wheel running have higher MOR mRNA expression in the nucleus accumbens than rats bred for low voluntary wheel running, indicating that MOR may be involved in the long-term motivation for exercise (Ruegsegger et al, 2015). Taken together, these preclinical results suggest that higher levels of exercise may produce greater compensatory responses within the MOR system.

HIIT exercise changes the radiotracer kinetics of $\left[{ }^{11} \mathrm{C}\right]$ carfentanil in a manner that poses challenges to quantitative modeling of receptor binding. Specifically, the radioactivity concentration was much lower after HIIT in all brain regions than after rest (Figure 3), suggesting altered peripheral distribution of the radiotracer after exercise (eg, due to vasodilatation, increased metabolism or excretion). The kinetic model compensates for such alterations, because specific binding in the target regions is compared with nondisplaceable binding in the receptor-free reference region. Nevertheless, some regions also showed decreased R1 values after HIIT, suggesting a potential effect of exercise on the relative delivery of the radiotracer to the brain. While this confounding factor cannot be definitively ruled out on the basis of current data, it is unlikely that decreased blood flow would explain decreased $B P_{\mathrm{ND}}$ after HIIT. First, previous simulations (Endres et al, 2003; Frost et al, 1989; Liberzon et al, 2002) have demonstrated that reference tissue modeling of specific binding is relatively insensitive to changes in cerebral blood flow. Second, decreased radioligand retention after HIIT was most pronounced during later phases of the study (Figure 3c), when blood flow no longer plays any substantial role for radioligand kinetics, suggesting that change in specific binding is more likely to explain this phenomenon. Taken together, it is unlikely that altered radioligand delivery confounded our finding of decreased
$B P_{\mathrm{ND}}$ after HIIT, but this matter cannot be resolved using current data. Future studies with simultaneous $\left[{ }^{11} \mathrm{C}\right]$ carfentanil PET and perfusion MRI could resolve the issue.

Some additional issues remain to be examined in future studies. First, females and males may have differential neurochemical responses to exercise (Heijnen et al, 2015) and different opioid responses to pain (Zubieta et al, 2002). Thus, the current results are not necessarily generalizable to females. Second, we did not assess the changes in affect while the subjects were recovering from exercise (during the PET studies). Affect fluctuates continuously and dynamically, and although high-intensity exercise increases negativity immediately after exercise, a shift towards positivity tends to occur during recovery of 10-20 min (Hall et al, 2002). Finally, we recruited recreationally active individuals with various sports backgrounds (from yoga to crossfit and martial arts). Future studies should determine whether similar MOR responses occur in competitive endurance athletes (such as runners, cyclists, or triathletes) and completely sedentary individuals after acute exercise, and even in subjects who dislike or even hate exercise, and, further, whether long-term repeated exercise sessions also modulate MOR.

We conclude that high-intensity interval training triggers opioid release, as evidenced by significant reductions in MOR availability, in key brain regions related to reward and pain processing. A reduction in MOR availability correlated with measures of negative emotionality. In contrast, moderate-intensity exercise did not impact on MOR availability, although it did elicit a positive mood and euphoria, which correlated with reductions in MOR availability. Endogenous opioid release thus depends on the intensity of exercise and on the concomitant affective changes. MOR action modulates positive emotionality after moderately intensive exercise, and negative emotionality or pain after highly intensive exercise. These effects may contribute significantly to the development and maintenance of exercise motivation in humans.

\section{FUNDING AND DISCLOSURE}

This study was financially supported by the Academy of Finland (grants 251125, 251399, 256470, 265917, 281440, 283319, and 304385), the Ministry of Education of the State of Finland, the Paavo Nurmi Foundation, the Finnish Cultural Foundation, the Novo Nordisk Foundation, the Paulo Foundation, the Turku Collegium for Science and Medicine, and the Hospital District of Southwest Finland, and the Medical Imaging Centre of Southwest Finland. TS was supported financially by the University of Turku Doctoral Programme of Clinical Research and by personal grants from the Juho Vainio Foundation, the Paavo Nurmi Foundation, the Instrumentarium Science Foundation, and the Veritas Foundation. LT received a research grant from the Sigrid Juselius Foundation. JJT was supported by a personal grant from the Finnish Cultural Foundation. The authors declare no conflict of interest.

\section{ACKNOWLEDGMENTS}

We thank the study participants and the personnel of Turku PET Centre, especially research technician Mikko Koivumäki, 
for excellent assistance during the study. We thank physicist Jarmo Teuho for PET image reconstruction and Jukka Kapanen for $\mathrm{VO}_{2 \max }$ determinations.

\section{REFERENCES}

Aaltonen S, Leskinen T, Morris T, Alen M, Kaprio J, Liukkonen J et al (2012). Motives for and barriers to physical activity in twin pairs discordant for leisure time physical activity for 30 years. Int J Sports Med 33: 157-163.

Aberg MAI, Pedersen NL, Torén K, Svartengren M, Bäckstrand B, Johnsson $\mathrm{T}$ et al (2009). Cardiovascular fitness is associated with cognition in young adulthood. Proc Natl Acad Sci USA 106: 20906-20911.

Arida RM, Gomes da Silva S, de Almeida AA, Cavalheiro EA, Zavala-Tecuapetla C, Brand S et al (2015). Differential effects of exercise on brain opioid receptor binding and activation in rats. J Neurochem 132: 206-217.

Bartlett JD, Close GL, MacLaren DPM, Gregson W, Drust B, Morton JP (2011). High-intensity interval running is perceived to be more enjoyable than moderate-intensity continuous exercise: implications for exercise adherence. J Sports Sci 29: 547-553.

Boecker H, Sprenger T, Spilker ME, Henriksen G, Koppenhoefer M, Wagner KJ et al (2008). The runner's high: opioidergic mechanisms in the human brain. Cereb Cortex 18: 2523-2531.

Bradley MM, Lang PJ (1994). Measuring emotion: the selfassessment manikin and the semantic differential. J Behav Ther Exp Psychiatry 25: 49-59.

Chartoff EH, Connery HS (2014). It's MORe exciting than mu: crosstalk between mu opioid receptors and glutamatergic transmission in the mesolimbic dopamine system. Front Pharmacol 5: 116.

Colasanti A, Searle GE, Long CJ, Hill SP, Reiley RR, Quelch D et al (2012). Endogenous opioid release in the human brain reward system induced by acute amphetamine administration. Biol Psychiatry 72: 371-377.

Colcombe SJ, Kramer AF, Erickson KI, Scalf P, McAuley E, Cohen NJ et al (2004). Cardiovascular fitness, cortical plasticity, and aging. Proc Natl Acad Sci USA 101: 3316-3321.

de Oliveira MSR, da Silva Fernandes MJ, Scorza FA, Persike DS, Scorza CA, da Ponte JB et al (2010). Acute and chronic exercise modulates the expression of MOR opioid receptors in the hippocampal formation of rats. Brain Res Bull 83: 278-283.

Decker ES, Ekkekakis P (2017). More efficient, perhaps, but at what price? Pleasure and enjoyment responses to high-intensity interval exercise in low-active women with obesity. Psychol Sport Exerc 28: 1-10.

Dietrich A, McDaniel WF (2004). Endocannabinoids and exercise. Br J Sports Med 38: 536-541.

Dishman RK, O’Connor PJ (2009). Lessons in exercise neurobiology: the case of endorphins. Ment Health Phys Act 2: 4-9.

Duerden EG, Albanese M-C (2013). Localization of pain-related brain activation: a meta-analysis of neuroimaging data. Hum Brain Mapp 34: 109-149.

Ekkekakis P, Parfitt G, Petruzzello SJ (2011). The pleasure and displeasure people feel when they exercise at different intensities. port Med 41: 641-671.

Endres CJ, Bencherif B, Hilton J, Madar I, Frost JJ (2003). Quantification of brain mu-opioid receptors with $\left[{ }^{11} \mathrm{C}\right]$ carfentanil: reference-tissue methods. Nucl Med Biol 30: 177-186.

Frost JJ, Douglass KH, Mayberg HS, Dannals RF, Links JM, Wilson AA et al (1989). Multicompartmental analysis of $\left[{ }^{11} \mathrm{C}\right]$-carfentanil binding to opiate receptors in humans measured by positron emission tomography. J Cereb Blood Flow Metab 9: 398-409.

Fuss J, Steinle J, Bindila L, Auer MK, Kirchherr H, Lutz B et al (2015). A runner's high depends on cannabinoid receptors in mice. Proc Natl Acad Sci USA 112: 13105-13108.
Goldfarb AH, Jamurtas AZ (1997). Beta-endorphin response to exercise. An update. Sports Med 24: 8-16.

Gunn RN, Lammertsma AA, Hume SP, Cunningham VJ (1997). Parametric imaging of ligand-receptor binding in PET using a simplified reference region model. Neuroimage 6: 279-287.

Haber SN, Knutson B (2010). The reward circuit: linking primate anatomy and human imaging. Neuropsychopharmacology 35: $4-26$.

Hall EE, Ekkekakis P, Petruzzello SJ (2002). The affective beneficence of vigorous exercise revisited. Br J Health Psychol 7: 47-66.

Heijnen S, Hommel B, Kibele A, Colzato LS (2015). Neuromodulation of aerobic exercise-a review. Front Psychol 6: 1890.

Henriksen G, Willoch F (2008). Imaging of opioid receptors in the central nervous system. Brain 131: 1171-1196.

Hirvonen J, Aalto S, Hagelberg N, Maksimow A, Ingman K, Oikonen $\mathrm{V}$ et al (2009). Measurement of central mu-opioid receptor binding in vivo with $\mathrm{PET}$ and $\left[{ }^{11} \mathrm{C}\right]$ carfentanil: a testretest study in healthy subjects. Eur J Nucl Med Mol Imaging 36: $275-286$.

Jung ME, Bourne JE, Little JP (2014). Where Does HIT Fit? An examination of the affective response to high-intensity intervals in comparison to continuous moderate- and continuous vigorous-intensity exercise in the exercise intensity-affect continuum. PLoS ONE 9: e114541.

Kanning M, Schlicht W (2010). Be active and become happy: an ecological momentary assessment of physical activity and mood. J Sport Exerc Psychol 32: 253-261.

Kilpatrick M, Kraemer R, Bartholomew J, Acevedo E, Jarreau D (2007). Affective responses to exercise are dependent on intensity rather than total work. Med Sci Sports Exerc 39: 1417-1422.

Leknes S, Tracey I (2008). A common neurobiology for pain and pleasure. Nat Rev Neurosci 9: 314-320.

Liberzon I, Zubieta JK, Fig LM, Phan KL, Koeppe RA, Taylor SF (2002). $\mu$-Opioid receptors and limbic responses to aversive emotional stimuli. Proc Natl Acad Sci 99: 7084-7089.

Mazzeschi C, Piana N, Capezzali D, Mommi A, Aiello C, Gatti M et al (2014). The impact of strenuous group physical activity on mood states, personal views, body composition, and markers of myocardial damage in overweight/obese adults: the 'Step-by-step Italy's coast to coast' trek. Biomed Res Int 2014: 854129.

Mick I, Myers J, Ramos AC, Stokes PRA, Erritzoe D, Colasanti A et al (2016). Blunted endogenous opioid release following an oral amphetamine challenge in pathological gamblers. Neuropsychopharmacology 41: 1742-1750.

Mick I, Myers J, Stokes PRA, Erritzoe D, Colasanti A, Bowden-Jones $\mathrm{H}$ et al (2014). Amphetamine induced endogenous opioid release in the human brain detected with $\left[{ }^{11} \mathrm{C}\right]$ carfentanil PET: replication in an independent cohort. Int J Neuropsychopharmacol 17: 2069-2074.

Milanović Z, Sporiš G, Weston M (2015). Effectiveness of high-intensity interval training (HIT) and continuous endurance training for $\mathrm{VO}_{2 \max }$ improvements: a systematic review and meta-analysis of controlled trials. Sports Med 45: 1469-1481.

Morgan WP (1985). Affective beneficence of vigorous physical activity. Med Sci Sports Exerc 17: 94-100.

Nummenmaa L, Tuominen L (2017). Opioid system and human emotions. Br J Pharmacol, doi:10.1111/bph.13812.

Oliveira BRR, Deslandes AC, Santos TM (2015). Differences in exercise intensity seems to influence the affective responses in self-selected and imposed exercise: a meta-analysis. Front Psychol 6: 1105.

Ramos JS, Dalleck LC, Tjonna AE, Beetham KS, Coombes JS (2015). The impact of high-intensity interval training versus moderateintensity continuous training on vascular function: a systematic review and meta-analysis. Sports Med 45: 679-692. 
Ruegsegger GN, Toedebusch RG, Will MJ, Booth FW (2015). Mu opioid receptor modulation in the nucleus accumbens lowers voluntary wheel running in rats bred for high running motivation. Neuropharmacology 97: 171-181.

Saanijoki T, Nummenmaa L, Eskelinen J-J, Savolainen AM, Vahlberg T, Kalliokoski KK et al (2015). Affective responses to repeated sessions of high-intensity interval training. Med Sci Sports Exerc 47: 2604-2611.

Saarimäki H, Gotsopoulos A, Jääskeläinen IP, Lampinen J, Vuilleumier P, Hari R et al (2016). Discrete neural signatures of basic emotions. Cereb Cortex 26: 2563-2573.

Schwarz L, Kindermann W (1992). Changes in beta-endorphin levels in response to aerobic and anaerobic exercise. Sport Med 13: 25-36.

Scott DJ, Stohler CS, Koeppe RA, Zubieta J-K (2007). Time-course of change in $\left[{ }^{11} \mathrm{C}\right]$ carfentanil and $\left[{ }^{11} \mathrm{C}\right]$ raclopride binding potential after a nonpharmacological challenge. Synapse 61: 707-714.

Scully D, Kremer J, Meade MM, Graham R, Dudgeon K (1998). Physical exercise and psychological well being: a critical review. Br J Sports Med 32: 111-120.

Sgherza AL, Axen K, Fain R, Hoffman RS, Dunbar CC, Haas F (2002). Effect of naloxone on perceived exertion and exercise capacity during maximal cycle ergometry. J Appl Physiol 93: 2023-2028.
Titeler M, Lyon RA, Kuhar MJ, Frost JF, Dannals RF, Leonhardt S et al (1989). Mu opiate receptors are selectively labelled by $\left[{ }^{3} \mathrm{H}\right]$ carfentanil in human and rat brain. Eur J Pharmacol 167: 221-228.

Trezza V, Damsteegt R, Achterberg EJM, Vanderschuren LJMJ (2011). Nucleus accumbens $\mu$-opioid receptors mediate social reward. J Neurosci 31: 6362-6370.

Wahl P, Mathes S, Köhler K, Achtzehn S, Bloch W, Mester J (2013). Acute metabolic, hormonal, and psychological responses to different endurance training protocols. Horm Metab Res 45: 827-833.

Watson D, Clark LA, Tellegen A (1988). Development and validation of brief measures of positive and negative affect: the PANAS scales. J Pers Soc Psychol 54: 1063-1070.

Weston KS, Wisløff U, Coombes JS (2014). High-intensity interval training in patients with lifestyle-induced cardiometabolic disease: a systematic review and meta-analysis. Br J Sports Med 48: 1227-1234.

Yeung RR (1996). The acute effects of exercise on mood state. J Psychosom Res 40: 123-141.

Zubieta J-K, Smith YR, Bueller JA, Xu Y, Kilbourn MR, Jewett DM et al (2002). $\mu$-opioid receptor-mediated antinociceptive responses differ in men and women. J Neurosci 22: 5100-5107.

Zubieta JK, Smith YR, Bueller JA, Xu Y, Kilbourn MR, Jewett DM et al (2001). Regional mu opioid receptor regulation of sensory and affective dimensions of pain. Science 293: 311-315.

Supplementary Information accompanies the paper on the Neuropsychopharmacology website (http://www.nature.com/npp) 